\title{
IMPROVED SNR ESTIMATION FOR BPSK AND QPSK SIGNALS
}

\author{
Ayesha ljaz, Adegbenga B. Awoseyila and Barry G. Evans \\ Centre for Communication Systems Research (CCSR), University of Surrey, \\ Guildford GU2 7XH, U.K. \\ a.ijaz@surrey.ac.uk, a.awoseyila@surrey.ac.uk,b.evans@surrey.ac.uk
}

A novel non-data-aided SNR estimation technique for BPSK and QPSK modulations in complex additive white Gaussian noise (AWGN) is proposed. It uses the constant amplitude property of in-phase and quadrature components of these modulations to achieve an improved performance. Its complexity is shown to be lower while its accuracy supersedes the popular moments-based estimator, approaching closely to the CRLB.

Introduction: Signal-to-noise ratio (SNR) is an important measure of the channel quality in many modern wireless communication systems. An accurate estimate of SNR is required for various applications such as link adaptation [1] and iterative decoding [2]. SNR estimation techniques can broadly be divided into two categories: data-aided (DA) and non-data-aided (NDA). Although DA estimators tend to be more accurate, NDA estimators have increased relevance when transmitted data are unknown, as is usually the case for applications which require frequent SNR estimates. Moreover, they are more bandwidth efficient since they do not require training symbols (pilots). Various NDA SNR estimation methods have been proposed for PSK signals in complex AWGN. In [3], the moments-based estimator (second- and fourth-order: $\left.M_{2} M_{4}\right)$ is reviewed and shown to achieve the Cramer-Rao lower 
bound (CRLB) [4] for a large sample size and at very high SNR. However, its performance degrades with respect to the CRLB as the sample size reduces. This constitutes a disadvantage for fast link adaptation where small observation windows are more appropriate. In [5], four SNR estimation algorithms using the absolute values of in-phase and quadrature components of the received QPSK signal are proposed, wherein the best performance shows some improvement over $M_{2} M_{4}$ for a moderate sample size and at SNR $>5 \mathrm{~dB}$. The method proposed in [6] is based on the data-aided ML approach [3], wherein modulation is removed by taking the $M^{\text {th }}$ power of the received signal. However, this process introduces a noise penalty $(\sim 12 \mathrm{~dB}$ for QPSK) which degrades accuracy. The envelope-based estimator in [7] achieves the same accuracy as $M_{2} M_{4}$ while the iterative bias compensation estimator in [8] achieves a reduced variance in the low SNR region. However, it is computationally intensive due to many iterations needed to achieve such accuracy.

We present a novel NDA SNR estimation technique for BPSK and QPSK based on the constant amplitude property of their in-phase and quadrature components. Its improved performance over the $M_{2} M_{4}$ estimator at moderate/high SNR is explained by mathematical derivations and verified by computer simulations.

System model: Let $S_{I k}$ and $S_{Q k}, k=1,2, \ldots, L$ be the in-phase and quadrature components of QPSK symbols transmitted over an AWGN channel. The signal components are assumed to be independent and identically distributed (i.i.d) discrete random variables, taking values in the set $\{a,-a\}$ with equal 
probability. Assuming one complex sample is taken for each of the $L$ symbols transmitted and that carrier synchronization exists, the $k^{\text {th }}$ received signal $Z_{k}$ can be described as:

$$
\begin{gathered}
X_{k}=S_{I k}+n_{I k} \\
Y_{k}=S_{Q k}+n_{Q k}
\end{gathered}
$$

where $X_{k}$ and $Y_{k}$ represent the in-phase and quadrature components of $Z_{k}$ respectively, while $n_{I k}$ and $n_{Q k}$ represent the in-phase and quadrature components of noise which are taken to be zero-mean i.i.d. Gaussian random variables, with variances $\sigma_{I}^{2}=\sigma_{Q}^{2}=\sigma^{2}$, respectively.

The SNR of the received signal is given by:

$$
\rho=\frac{S}{N}=\frac{S_{I}^{2}+S_{Q}^{2}}{E\left\{n_{I}^{2}+n_{Q}^{2}\right\}}=\frac{a^{2}+a^{2}}{\sigma_{I}^{2}+\sigma_{Q}^{2}}=\frac{2 a^{2}}{2 \sigma^{2}}
$$

where $S$ is the signal power and $N$ is the noise power.

Assuming the same conditions as above for BPSK modulation with $S_{I k} \in\{-a, a\}$ and $S_{Q k}=0$, signal power equal to $a^{2}$ is employed in (3).

The second order moment $\left(M_{2}\right)$ of the received signal is is given as:

$$
\begin{aligned}
M_{2}= & E\left\{\left|Z_{k}\right|^{2}\right\}=E\left\{X_{k}{ }^{2}\right\}+E\left\{Y_{k}{ }^{2}\right\} \\
& =E\left\{S_{I k}^{2}+n_{I k}^{2}+2 S_{I k} n_{I k}\right\}+E\left\{S_{Q k}^{2}+n_{Q k}^{2}+2 S_{Q k} n_{Q k}\right\}
\end{aligned}
$$


Since the noise and signal components are independent, $M_{2}$ is shown to be equal to the sum of signal and noise power as follows:

$$
\begin{aligned}
M_{2} & =E\left\{S_{I k}^{2}\right\}+E\left\{n_{I k}^{2}\right\}+E\left\{S_{Q k}^{2}\right\}+E\left\{n_{Q k}^{2}\right\} \\
& =a^{2}+\sigma_{I}^{2}+a^{2}+\sigma_{Q}^{2}=2 a^{2}+2 \sigma^{2}=S+N
\end{aligned}
$$

Therefore, the SNR can be estimated as follows:

$$
\hat{\rho}=\frac{\hat{S}}{\hat{M}_{2}-\hat{S}}
$$

where $\hat{M}_{2}=\frac{1}{L} \sum_{k=1}^{L}\left|Z_{k}\right|^{2}=\frac{1}{L} \sum_{k=1}^{L}\left[X_{k}^{2}+Y_{k}^{2}\right]$

Proposed Method: For BPSK and QPSK modulations in complex AWGN, it is observed that the absolute values of the in-phase and quadrature components of the received signal have a close relationship with the signal power, since these components have a constant amplitude in the transmit signal. Consider a received QPSK signal as presented in the system model. Since $\left|S_{I k}\right|=\left|S_{Q k}\right|=a, k=1,2, \ldots, L$,

$$
E\left\{\left|S_{I k}\right|\right\}=E\left\{\left|S_{Q k}\right|\right\}=a
$$

The absolute values of the in-phase and quadrature components of the received signal can be expressed as:

$$
\left|X_{k}\right|=\left|S_{I k}+n_{I k}\right|
$$


$\left|Y_{k}\right|=\left|S_{Q k}+n_{Q k}\right|$

Assuming that $\left|n_{k}\right|<\left|S_{k}\right|$ (which is usually valid at moderate/high SNR):

$$
\begin{array}{ll}
\left|X_{k}\right|=\left|S_{I k}\right|+n_{I k} & \left(X_{k}>0\right) \\
\left|X_{k}\right|=\left|S_{I k}\right|-n_{I k} & \left(X_{k}<0\right) \\
\left|Y_{k}\right|=\left|S_{Q k}\right|+n_{Q k} & \left(Y_{k}>0\right) \\
\left|Y_{k}\right|=\left|S_{Q k}\right|-n_{Q k} & \left(Y_{k}<0\right)
\end{array}
$$

Using (7) and (10) -(13) and given that the noise components are zero-mean,

$$
\begin{aligned}
& E\left\{\left|X_{k}\right|\right\}=E\left\{\left|S_{I k}\right|\right\} \pm E\left\{n_{I k}\right\}=E\left\{\left|S_{I k}\right|\right\}=a \\
& E\left\{\left|Y_{k}\right|\right\}=E\left\{\left|S_{Q k}\right|\right\} \pm E\left\{n_{Q k}\right\}=E\left\{\left|S_{Q k}\right|\right\}=a
\end{aligned}
$$

Hence, the transmitted signal power can be determined using the mean of the absolute values of in-phase and quadrature components of the received signal, i.e.

$$
S=E\left\{\left|X_{k}\right|\right\}^{2}+E\left\{\left|Y_{k}\right|\right\}^{2}=a^{2}+a^{2}=2 a^{2}
$$

Consequently, our proposed method estimates the transmitted signal power using the samples of the received signal as follows: 
$\hat{S}_{\text {proposed }}=\frac{1}{L^{2}}\left[\left(\sum_{k=1}^{L}\left|X_{k}\right|\right)^{2}+\left(\sum_{k=1}^{L}\left|Y_{k}\right|\right)^{2}\right]$

The moments-based method estimates the transmitted signal power as follows [3]:

$\hat{S}_{M_{2} M_{4}}=\sqrt{2 \hat{M}_{2}^{2}-\hat{M}_{4}}$

where $\hat{M}_{4}=\frac{1}{L} \sum_{k=1}^{L}\left|Z_{k}\right|^{4}$

In contrast to the $M_{2} M_{4}$ estimator which makes use of the constant envelope property of the transmitted signal, our proposed estimator takes full advantage of the constant amplitude property of in-phase and quadrature components of the signal, and is therefore able to achieve improved accuracy when the estimator assumptions are valid. The proposed method also has a lower complexity than $M_{2} M_{4}$ as shown in Table 1, wherein it is seen that only three real multiplications are required in the computation of $\hat{S}_{\text {proposed }}$ while the number of real multiplications needed to compute $\hat{M}_{4}$ increases linearly with the observation window $L$. For a moderate sample size of $L=64$, this translates into 193 real multiplications.

Computer simulations: Computer simulations (10,000 trials) were performed to verify the performance of the proposed method in comparison to $M_{2} M_{4}$ and the best estimator in [5], using $L=64$ QPSK/BPSK symbols in complex 
AWGN. Fig. 1 shows the bias of the estimators in terms of mean SNR estimate, wherein it can be seen that all the estimators exhibit very low bias at moderate/high SNR. The proposed method has a low bias for BPSK at low SNR while $M_{2} M_{4}$ maintains this for both BPSK and QPSK. The increasing bias noticed for the proposed method at low SNR is due mainly to an increasing departure from the assumptions used to derive it. Fig. 2 shows the accuracy of the estimators in terms of mean square error (MSE), normalised to the true SNR as defined in [3], wherein the CRLB (DA) is also shown for comparison purposes. For BPSK, the proposed method outperforms $M_{2} M_{4}$ at all values of SNR, with its MSE approaching closely to the CRLB at SNR > 3dB. In the case of QPSK, the proposed method maintains its superiority over $M_{2} M_{4}$ for SNR $>3 \mathrm{~dB}$, while approaching closely to the CRLB at SNR $>7 \mathrm{~dB}$.

Conclusions: We have proposed a novel NDA SNR estimator for BPSK and QPSK modulations and it is shown to be better than the popular momentsbased estimator in terms of lower computational complexity and improved accuracy at SNR regions of interest. Furthermore, its accuracy approaches closely to the CRLB at moderate SNR.

Acknowledgment: This work was supported in part by the EC FP6 SatNEx-II Project (IST-027393). 


\section{References}

1. Chung T. S., and Goldsmith A. J., "Degrees of freedom in adaptive modulation: a unified view," IEEE Trans. Commun., vol. 49, no. 9, pp. 1561 - 1571, Sep. 2001.

2. Summers T. A., and Wilson S. G., "SNR mismatch and online estimation in turbo decoding," IEEE Trans. Commun., vol. 46, no. 4, pp. 421 - 423, Apr. 1998.

3. Pauluzzi D. R., and Beaulieu N. C., "A comparison of SNR estimation techniques for the AWGN channel," IEEE Trans. Commun., vol. 48, no. 10, pp. 1681 - 1691, Oct. 2000.

4. Alagha, N. S., "Cramer-Rao bounds of SNR estimates for BPSK and QPSK modulated signals," IEEE Commun. Lett., vol. 5, no. 1, pp. 10-12, Jan. 2001.

5. Beaulieu N. C., Toms A. S. , and Pauluzzi D. R., "Comparison of four SNR estimators for QPSK modulations," IEEE Commun. Lett., vol. 4, no. 2, pp. 43 - 45, Feb. 2000.

6. Xu H., and Zheng H., "The simple SNR estimation algorithm for MPSK signals," Proc. Intl. Conf. Sig. Process. (ICSP), vol. 2, pp. 1781 - 1785, Aug. - Sep. 2004.

7. Pei-jun Y. and Jian-ping A., "SNR estimation for constant envelope signals in AWGN channel," Proc. Intl. Conf. on Wireless Commun., Net. and Mobile Computing, vol. 1, pp. 525 - 528, Sep. 2005.

8. Bakkali M., Stéphenne A., and Affes S., "Iterative SNR estimation for MPSK modulation over AWGN channels," Proc. IEEE 64th Veh. Tech. Conf. (VTC), pp. 1 - 5, Sep. 2006. 


\section{Figure/Table captions:}

Table 1 Complexity comparison between proposed method and $\mathrm{M}_{2} \mathrm{M}_{4}$

Fig. 1 Mean of estimated SNR, $L=64$

Fig. 2 Normalised MSE of different estimators, $L=64$ 
Table 1

\begin{tabular}{|l|l|l|}
\hline Computation & $\hat{S}_{\text {proposed }}$ & $\hat{M}_{4}$ \\
\hline Real Multiplications & 3 & $3 L+1$ \\
\hline Real Additions & $2 L-1$ & $2 L-1$ \\
\hline
\end{tabular}


Figure 1

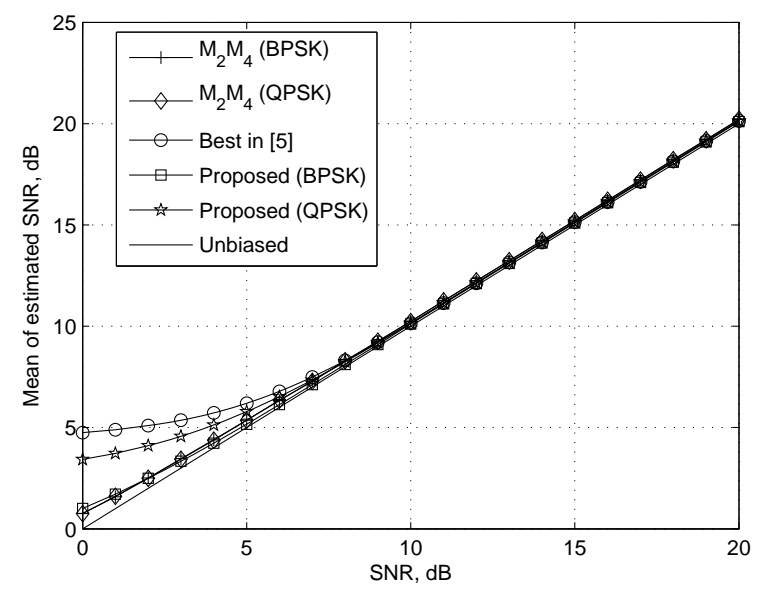


Figure 2

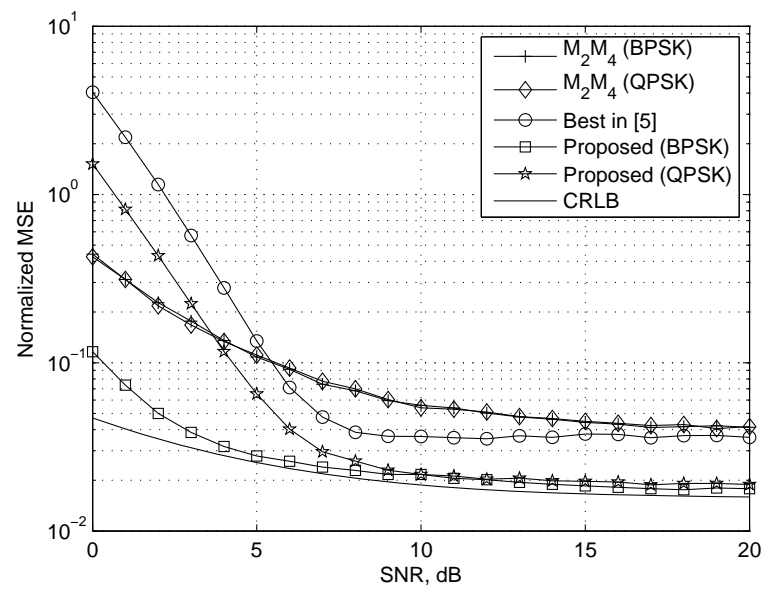

